\title{
For Huda Zoghbi, collaboration is the key to unlocking the secrets of neurobiology
}

$\ln$ Peter and Patricia Gruber Foundation awarded the 2011 Neuroscience Prize to Huda Zoghbi in recognition of her work uncovering the genetic and molecular causes of neurological disorders. Zoghbi, a professor at Baylor College of Medicine, is also the director of the new Jan and Dan Duncan Neurological Research Institute (NRI), which opened in late 2010 . The JCI recently spoke to Zoghbi about her accomplishments.

JCI: How did you get interested in pediatric neurology?

Zoghbi: I came to Baylor College of Medicine to be a pediatric cardiologist, and I didn't have neurology on my radar screen. But during my pediatric residency, I rotated in neurology with Dr. Marvin Fishman, and the whole experience of encountering a neurological problem and figuring it out based on a very good clinical history and exam fascinated me. It felt like detective work. Afterward, I still thought I was going to be a cardiologist, but a month away from it, I missed neurology very much, and I decided I had to come back and revisit it. For me, part of the appeal of neurology was that if you take the time to think through the problem, most of the time you can reach the right clinical and anatomical diagnosis.

JCI: You've made a number of remarkable discoveries, including identifying the genes that underlie Rett syndrome (methyl-CpGbinding protein $2[M E C P 2])$ and spinocerebellar ataxia type 1 (SCA1). What do you think has been your greatest accomplishment so far?

Zoghbi: I really think my greatest accomplishment has been putting together a team of wonderful trainees and working with great collaborators like Dr. Harry Orr, my long-time partner in our SCA1 studies. I've done some things myself, but we can only accomplish so much as individuals - my trainees are the ones who have made many of the discoveries that we're all excited about. But I feel the best is yet to come: when I can make a patient with Rett syndrome or SCA1 better, that will be the day I feel that I truly made a difference. I am holding out for that. We've learned so much since the discovery of the genes that we're well along on the path to finding a way to temper the disease course. Research really does pay off - as long as we're willing to be patient, and do the long and hard experiments.

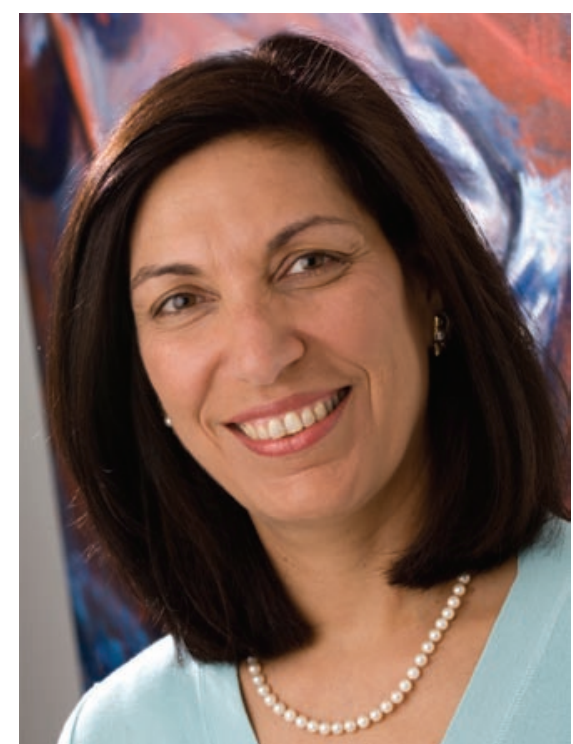

that the final behavioral phenotype might result from failure of neuronal homeostasis rather than a specific physiological change. This phenomenon has been observed in many autism spectrum disorders where either a gain or a loss of a genomic region results in an autism phenotype.

JCI: What is your vision for the NRI?

Zoghbi: My vision is to create an environment that will usher in the next generation of investigators to help us understand and develop treatments for childhood neurological diseases. I had to think about what I thought would create a change in diseaseoriented research. A key step was to build an environment that would enhance collaboration and that will support investigators by giving them resources and access to specialized core facilities.

JCI: What makes the NRI unique?

Zoghbi: It is - to my knowledge - the JCI: How has your study of Rett syndrome affected our understanding of autism?

Zoghbi: There are really many areas where the research on Rett has been informative about the causes and biology of autism. For example, we first discovered that mutations in MECP2 cause Rett syndrome but then we quickly learned that mutations in this gene cause other disorders. MECP2 is X-linked, so Rett girls have half of their cells with the $\mathrm{X}$ chromosome carrying the normal MECP2 allele active and the other half with the $\mathrm{X}$ chromosome carrying the mutant allele active. We and others found that mutations in this gene can cause some cases of isolated autism. This happens when girls have more cells with the $\mathrm{X}$ chromosome carrying the normal MECP2 allele as the active one. Such favorable $\mathrm{X}$ chromosome activation pattern leads to milder symptoms. So if $80 \%$ of their cells express the normal copy, those patients tend to present with clinical autism rather than Rett syndrome.

We've also learned that both the loss and the gain of this gene can lead to autism phenotypes, which suggests that having the right amount of this protein is really critical for normal neurological function. That's really interesting because although loss and gain of the gene cause opposite morphological and electrophysiological phenotypes, they actually cause similar and overlapping behavioral phenotypes. These data suggest first institute dedicated to basic research on childhood neurological disorders and neurodevelopment. It's a 13-floor building totally dedicated to interdisciplinary basic research. Neurological diseases are complicated, and no one can solve them if they only have two or three skill sets. The faculty come from diverse fields - biochemistry, genetics, cell biology, pathology, the physical sciences, bioinformatics, etc., and they are encouraged to collaborate and interact. If there's one thing I've learned from doing diseaseoriented research, it's that you really have to come at it from multiple disciplines, and examine many aspects of the disease process, not just the gene, the behavior, or the physiology. The NRI is designed to increase opportunities for interactions.

JCI: What does winning the Gruber Foundation Neuroscience Prize mean to you?

Zoghbi: It is a great honor. It's really a tribute to my mentors, my collaborators, my trainees, my husband, and family. Beyond that, I truly appreciate the foundation's acknowledgement of the importance of disease-oriented research. I'm hoping through this additional support that we can continue to push the research forward. It's certainly energized the lab, and energized the institute, in ways you cannot imagine. I tell everyone in the lab, if I can do it, you can do it.

\section{Kathryn Claiborn}

